\title{
Ressonância magnética estrutural nos transtornos afetivos
}

\author{
Roberto B Sassia e Jair C Soares ${ }^{b}$
}

\begin{abstract}
${ }^{a}$ Department of Psychiatry, Western Psychiatric Institute and Clinic, University of Pittsburgh School of Medicine. Pittsburgh, PA, USA e Departmento de Psiquiatria do Instituto de Psiquiatria da Faculdade de Medicina da Universidade de São Paulo. São Paulo, SP, Brasil. 'bepartment of Psychiatry, Western Psychiatric Institute and Clinic, University of Pittsburgh School of Medicine. Pittsburgh, PA, USA
\end{abstract}

\section{Introdução}

A busca de um substrato anatômico para os transtornos afetivos avançou consideravelmente nas últimas duas décadas graças a técnicas mais precisas e refinadas de novas metodologias de neuroimagem. Mais recentemente, a ressonância magnética nuclear (RMN) suplantou o uso da tomografia computadorizada (TC) nos estudos em psiquiatria, por não envolver radiação ionizante, gerar imagens de alta resolução anatômica e permitir medidas volumétricas mais acuradas de diversas regiões e estruturas do sistema nervoso central. Em anos recentes, estudos comparando medidas anatômicas cerebrais entre grupos de voluntários normais e pacientes com transtornos afetivos primários têm demonstrado algumas alterações significativas de potencial relevância para a patofisiologia desses transtornos, tanto regionais quanto generalizadas, questionando a clássica divisão entre doenças psiquiátricas "funcionais" e "orgânicas".

Baseando-se nesses achados preliminares, pode-se postular um modelo para os circuitos neuronais responsáveis pela expressão e pela regulação das emoções (Figura), que podem possivelmente estar envolvidos nos transtornos afetivos, abrangendo conexões entre o córtex pré-frontal, o tálamo, o complexo amígdala-hipocampo, os gânglios da base e, possivelmente, o cerebelo. ${ }^{1}$ Esse modelo permite testar novas hipóteses quanto às bases anatômicas dos transtornos afetivos. No presente artigo, apresentamos uma sucinta revisão dos estudos de neuroimagem estrutural nos transtornos afetivos primários, enfatizando as semelhanças e diferenças encontradas entre pacientes com transtorno afetivo bipolar e unipolar.

Figura 1 - Modelo neuroanatômico de regulação do humor*

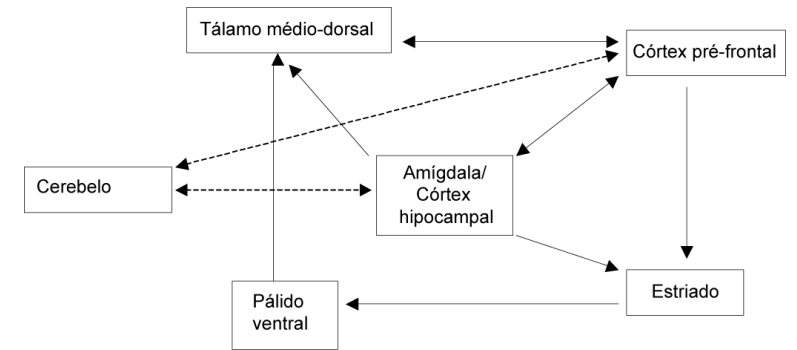

Conexões entre estruturas subcorticais, cerebelo e áreas do córtex pré-frontal compõem os circuitos-chave na expressão e regulação das emoções. Anormalidades ou disfunções nestes circuitos estariam envolvidas nos transtornos afetivos.

*Adaptado de Soares \& Mann, 1997.

\section{Alterações generalizadas}

De maneira geral, algumas alterações encefálicas generalizadas têm sido relatadas nos transtornos afetivos. Didaticamente, pode-se dividir os achados em três grupos.

\section{Atrofia global}

Diversos índices têm sido utilizados para avaliar atrofia cerebral nos estudos de neuroimagem, entre eles largura dos sulcos, volume cerebral total e volume ventricular. Estudos mais recentes com RMN têm utilizado técnicas semi-automatizadas ou automatizadas, e portanto mais precisas, para calcular os volumes de substância branca ou cinzenta no cérebro humano. Diferenças entre as técnicas utilizadas para as medidas volumétricas, assim como a inclusão de bipolares e unipolares num mesmo grupo de pacientes, podem ter contribuído para a falta de consenso entre os resultados de diversos estudos. Quanto ao volume dos ventrículos, por exemplo, vários estudos controlados com TC e RMN não demonstraram alterações entre pacientes e controles em relação aos ventrículos laterais. ${ }^{2,3}$ Outros estudos, entretanto, encontraram um aumento do volume dos ventrículos laterais em bipolares ${ }^{4} \mathrm{e}$ unipolares. ${ }^{5}$ Apesar de não estar presente em todos os pacientes, o aumento de volume dos ventrículos laterais parece ocorrer mais comumente nos pacientes bipolares do que nos unipolares, embora esse achado não seja tão consistente quanto nos pacientes com esquizofrenia. Além disso, ventriculomegalia em pacientes unipolares parece ser mais comum na população geriátrica do que em adultos jovens, mesmo comparando com controles pareados para idade. ${ }^{5}$ Aumento de volume do terceiro ventrículo também parece ser mais comumente encontrado em pacientes bipolares ${ }^{6}$ do que unipolares. ${ }^{7}$

$\mathrm{O}$ exato significado neuropatológico do aumento dos ventrículos nos transtornos afetivos ainda não é claro. De maneira geral, a maioria dos estudos não demonstrou atrofia das regiões periventriculares ou diencefálicas nesses pacientes. ${ }^{1}$ Da mesma forma, não há evidências conclusivas de atrofia cortical generalizada na maioria dos estudos envolvendo pacientes com transtornos afetivos. Vários estudos controlados usando medidas de atrofia sulcal, volume cerebral total ou substância cinzenta não demonstraram diferenças entre pacientes com transtornos afetivos e controles. ${ }^{89} \mathrm{Um}$ estudo controlado, com medidas semi-automatizadas de substância cinzenta e branca, foi realizado pelo grupo dos autores do 
presente artigo comparando pacientes com transtorno bipolar e voluntários saudáveis com menos de 60 anos.* Não foi encontrada diferença entre os grupos, embora os pacientes apresentassem uma correlação negativa entre idade e volume de substância cinzenta, que não foi encontrada nos controles. Esse achado sugere que, apesar de não apresentarem menor concentração de substância cinzenta que os voluntários normais, os pacientes bipolares podem sofrer uma diminuição da densidade de substância cinzenta com a idade de maneira mais pronunciada do que no envelhecimento normal. Entretanto, embora atrofia cerebral generalizada não tenha sido identificada na maioria dos estudos nos transtornos afetivos, uma metanálise dessa literatura ${ }^{10}$ demonstrou maior freqüência de atrofia cortical nos pacientes com transtorno de humor do que nos controles, embora menos pronunciada do que nos pacientes com esquizofrenia. Pode-se considerar que, se há atrofia generalizada nos transtornos afetivos, a magnitude é relativamente pequena, detectável apenas em estudos com grande número de pacientes e controles.

\section{Sinais hiperintensos em substância branca (SHSB)}

Um dos achados mais replicados na literatura em neuroimagem dos transtornos afetivos é a freqüência aumentada de pequenas lesões difusas em substância branca nos pacientes em relação aos controles. Essas lesões aparecem como sinais hiperintensos na RMN em T2, freqüentemente periventriculares, mas também são encontradas nos núcleos subcorticais. A etiopatologia dessas lesões não é clara, mas parecem ter sua origem ligada a distúrbios cerebrovasculares. SHSB foram consistentemente encontrados em pacientes unipolares ${ }^{7} \mathrm{e}$ bipolares. ${ }^{11}$ Aparentemente, são mais freqüentes no transtorno bipolar tipo I do que no tipo II e em unipolares idosos do que em adultos jovens. Maiores taxas de SHSB também são encontradas em familiares sem transtornos psiquiátricos de pacientes bipolares. ${ }^{12}$ SHSB também são observados com maior freqüência em outras patologias psiquiátricas, ou mesmo no envelhecimento normal. Ainda não está estabelecido se essas lesões poderiam representar processos degenerativos da doença afetiva ou se estariam relacionadas com o uso crônico de medicações. Essas lesões vasculares poderiam eventualmente interromper circuitos neuronais responsáveis pela regulação do humor e seriam causas de depressão secundária em populações específicas, como os idosos. Mais estudos são necessários para esclarecer a relevância desses achados nos transtornos afetivos primários.

\section{Assimetria inter-hemisférica}

O cérebro humano apresenta importante assimetria entre os hemisférios. Diversas estruturas e regiões cerebrais, em especial as ligadas com o desenvolvimento da linguagem, são maiores no hemisfério esquerdo do que no direito. Falhas na lateralização hemisférica têm sido relacionadas com maior vulnerabilidade a psicoses. Nesse sentido, um estudo de $\mathrm{RMN}^{13}$ demonstrou um "contínuo" de assimetria, com voluntários nor- mais, pacientes com transtornos afetivos e pacientes esquizofrênicos, respectivamente, em ordem decrescente de assimetria inter-hemisférica. Vários estudos avaliando o planum temporale em esquizofrenia demonstram perda da lateralização normal, mas os resultados não são tão claros no transtorno bipolar. ${ }^{14}$ Não há, até o momento, estudos controlados comparando assimetria hemisférica e sintomas psiquiátricos em pacientes com transtornos afetivos.

\section{Alterações localizadas}

\section{Lobo frontal}

Considerando o lobo frontal como um todo, alguns estudos demonstraram menor área ou densidade de substância cinzenta em pacientes unipolares ${ }^{7}$ e bipolares, ${ }^{15}$ consistente com o hipometabolismo frontal evidenciado em estudos funcionais nos transtornos afetivos. ${ }^{16}$ A maioria dos estudos considerou o córtex pré-frontal como uma região única, e vários estudos produziram resultados negativos. ${ }^{6}$ Em contraste com esses estudos, Drevets et $\mathrm{al}^{17}$ demonstraram uma redução volumétrica em uma sub-região específica do córtex pré-frontal, ventral ao joelho do corpo caloso, correspondente à área de Broadmann 24. Essa redução volumétrica no córtex pré-frontal sub-genual foi encontrada em pacientes deprimidos unipolares e bipolares com história familiar de transtorno afetivo. Pacientes com esquizofrenia, ou mesmo com transtorno afetivo, mas sem história familiar, não apresentavam alterações nessa região. Hipometabolismo e diminuição na densidade de glia no córtex sub-genual foram relatados por esse mesmo grupo nos transtornos afetivos com história familiar positiva. Redução no volume do córtex pré-frontal sub-genual esquerdo também foi encontrada por outros pesquisadores em pacientes com sintomas psicóticos durante o primeiro episódio afetivo, unipolar ou bipolar, mas somente nos indivíduos com história familiar positiva para transtornos de humor. ${ }^{18}$ Pacientes esquizofrênicos, em primeiro episódio, não apresentavam alterações volumétricas nessa região cortical, reforçando a hipótese de uma anormalidade cerebral específica nos pacientes unipolares e bipolares com história familiar positiva para transtornos afetivos.

\section{Lobo temporal}

Estudos relacionados a área ou volume do lobo temporal têm produzido resultados conflitantes. ${ }^{2,19}$ Por outro lado, estudos que examinaram estruturas temporais mediais têm gerado resultados mais consistentes. Atrofia hipocampal foi observada em pacientes deprimidos ${ }^{20}$ correlacionada com a duração do episódio depressivo. ${ }^{21}$ Esses achados sugerem que o estresse crônico pode levar à lesão neuronal, possivelmente pela ação dos elevados níveis de glucocorticóides sobre as células hipocampais. Aumento do volume da amígdala foi relatado no transtorno bipolar, ${ }^{22,23}$ em concordância com estudos funcionais demonstrando maior ativação da amígdala na depressão. Entretanto, outros estudos não encontraram alterações volumétricas em hipocampo ${ }^{22}$ ou em amígdala ${ }^{20}$ nos transtor-

*Brambilla P, Harenski K, Nicoletti M, Mallinger AG, Frank E, Kupfer DJ, et al. Differential effects of age on brain gray matter in bipolar patients and healthy individuals. Neuropsychobiol 2001. No prelo. 
nos afetivos. Diferentes técnicas de aquisição das imagens, ou mesmo discrepâncias quanto à delimitação anatômica das regiões a serem medidas, podem explicar a ausência de consenso entre os achados.

\section{Gânglios da base}

$\mathrm{O}$ envolvimento dos gânglios da base parece ser distinto entre o transtorno bipolar e o unipolar. Alguns estudos com pacientes bipolares demonstraram aumento de volume dos núcleos caudado ${ }^{8}$ e estriado, ${ }^{23}$ embora a maioria dos estudos não tenha encontrado alterações. ${ }^{6,9}$ Por outro lado, em pacientes unipolares as evidências parecem ser mais consistentes, com estudos controlados demonstrando redução de volume do caudado e do putamen. ${ }^{24,25}$ Esses achados preliminares necessitam ser replicados e ampliados para esclarecer o papel dos gânglios da base nos transtornos afetivos.

\section{Cerebelo}

O cerebelo, e mais especificamente o vermis, tem conexões eferentes e aferentes com várias regiões cerebrais envolvidas na cognição e na regulação do humor, como, por exemplo, a amígdala e o córtex pré-frontal. Em unipolares, atrofia cerebelar foi relacionada com gravidade dos sintomas depressivos e falha de resposta a antidepressivos. ${ }^{26}$ Atrofia cerebelar foi também encontrada em pacientes bipolares. ${ }^{27}$ Nesse último estudo, ${ }^{27}$ apenas os pacientes com múltiplos episódios afetivos apresentavam atrofia cerebelar. Dessa forma, as evidências sugerem que o envolvimento do cerebelo nos transtornos afetivos esteja relacionado com cronicidade e gravidade dos sintomas, talvez representando processos neurodegenerativos causados pela própria patologia ou pelo uso crônico de medicações.

\section{Corpo caloso}

Anormalidades na forma e volume do corpo caloso foram relatadas em estudos preliminares em pacientes com transtornos afetivos, ${ }^{28}$ sugerindo alterações em substância branca. Esses estudos iniciais, entretanto, não foram replicados com técnicas mais recentes.

\section{Tálamo}

Apesar da importância do tálamo nos circuitos neuronais envolvidos no controle e na expressão do humor, não há consenso quanto a alterações estruturais nos transtornos afetivos. Enquanto alguns estudos não encontraram diferenças significativas no volume talâmico entre pacientes e controles, ${ }^{15}$ outros relatam aumento ${ }^{23}$ ou diminuição $0^{29}$ no transtorno bipolar. Em unipolares os resultados também divergem. ${ }^{1}$

\section{Discussão}

De maneira geral, os estudos estruturais nos transtornos afetivos convergem para o envolvimento de regiões e circuitos encefálicos específicos. Mais do que um processo "funcional" difuso, os transtornos afetivos parecem estar relacionados com anormalidades em estruturas-chave representadas no modelo neuroanatômico proposto para regulação do humor (Figura). Alterações volumétricas em áreas específicas do córtex pré-frontal, do hipocampo, da amígdala, do cerebelo e, possivelmente, dos gânglios da base são encontradas mais freqüentemente nos pacientes com transtornos afetivos do que em controles saudáveis. É provável que diferentes processos patológicos incidam sobre as diversas regiões envolvidas na expressão emocional. Por exemplo, o córtex préfrontal sofre intensa maturação e "poda" sináptica durante a infância. Teoricamente, experiências emocionais e estímulos ambientais nesse período seriam capazes de influenciar a neuroarquitetura cortical, com resultado final no volume de substância cinzenta medido na RMN. Atrofia hipocampal e cerebelar, por outro lado, parecem estar envolvidas com múltiplos episódios afetivos e/ou com maior gravidade dos sintomas e podem representar alterações neurodegenerativas, reversíveis ou não. A redução de densidade de células da glia (com conseqüente redução volumétrica) no córtex pré-frontal sub-genual parece ocorrer apenas nos casos de transtorno afetivo com história familiar positiva, sugerindo uma alteração do neurodesenvolvimento de provável origem genética em ao menos uma parcela dos casos. Além disso, os estudos estruturais apontam para algumas semelhanças e diferenças entre os transtornos afetivos bipolar e unipolar. Aumento do volume do terceiro ventrículo e dos ventrículos laterais, perda da lateralização hemisférica e presença de SHSB parecem ser mais intensos no transtorno bipolar. $\mathrm{O}$ acometimento dos gânglios da base parece ser distinto entre as duas patologias e mais consistentemente relacionado com o transtorno unipolar. Atrofia hipocampal também tem sido relatada de forma mais consistente em pacientes unipolares. Por outro lado, o acometimento do cerebelo, aparentemente relacionado com a gravidade e a cronicidade dos sintomas afetivos, parece ser semelhante entre pacientes bipolares e unipolares. Essas são conclusões bastante preliminares e que precisam ser examinadas com cautela, devido à escassez de estudos comparando diretamente pacientes bipolares e unipolares com semelhantes características clínicas, como número de episódios afetivos prévios ou classes de medicações utilizadas.

Os estudos de neuroimagem estrutural sofrem várias falhas na identificação dos circuitos envolvidos nos transtornos afetivos. A primeira delas é inerente à própria técnica: circuitos que funcionem de maneira anormal, mas que mantenham a mesma densidade de células (ou seja, o mesmo volume final), não serão detectados. Para isso, existem as técnicas funcionais, como a espectroscopia por ressonância magnética, a tomografia por emissão de pósitrons (PET) ou a ressonância funcional, que de maneira geral vêm confirmando os achados estruturais. Deficiências no desenho de alguns dos estudos aqui revistos dificultam a interpretação dos achados. Entre as limitações, podemos citar o fato de muitos deles incluírem em um mesmo grupo pacientes bipolares e unipolares, ou em diferentes fases da doença - por exemplo, durante um episódio depressivo ou em eutimia. Há uma escassez de estudos de seguimento, necessários para verificar se as alterações encontradas são traço ou estado-dependentes. Perspectivas para estudos futuros devem incluir comparações de características clínicas específicas 
entre os grupos de pacientes, como por exemplo presença ou não de sintomas psicóticos ou história familiar positiva para transtornos afetivos. Estudos em familiares saudáveis de pacientes com transtornos de humor são necessários para investigar quais tipos de alterações estruturais conferem proteção ou vulnerabilidade à doença afetiva.
Trabalho realizado parcialmente com o apoio financeiro das linhas de pesquisa MH01736, MH29618 e MH30915 (NIMH) da Theodore and Vada Stanley Foundation, da National Alliance for Research in Schizophrenia and Affective Disorders (NARSAD), EUA; e da Fundação CAPES, Brasil, Processo BEX 0374/00-8.

\section{Referências}

1. Soares JC, Mann JJ. The anatomy of mood disorders - Review of structural neuroimaging studies. Biol Psychiatry 1997;41:86-106.

2. Harvey I, Persaud R, Ron MA, Baker G, Murray RM. Volumetric MRI measurements in bipolars compared with schizophrenics and healthy controls. Psychol Med 1994;24:689-99.

3. Risch SC, Lewine RJ, Kalin NH, Jewart RD, Risby ED, Candle JM, et al. Limbic-hypothalamic-pituitary-adrenal axis activity and ventricular-to-brain ratio studies in affective illness and schizophrenia. Neuropsychopharmacol 1992;6:95-100.

4. Andreasen NC, Swayze Vd, Flaum M, Alliger R, Cohen G. Ventricular abnormalities in affective disorder: clinical and demographic correlates. Am J Psychiatry 1990;147:893-900.

5. Wurthmann C, Bogerts B, Falkai P. Brain morphology assessed by computed tomography in patients with geriatric depression, patients with degenerative dementia, and normal control subjects. Psychiatry Res 1995;61:103-11.

6. Strakowski SM, Wilson DR, Tohen M, Woods BT, Douglass AW, Stoll AL. Structural brain abnormalities in first-episode mania. Biol Psychiatry 1993;33:602-9.

7. Coffey CE, Wilkinson WE, Weiner RD, Parashos IA, Djang WT, Webb MC, et al. Quantitative cerebral anatomy in depression. A controlled magnetic resonance imaging study. Arch Gen Psychiatry 1993;50:7-16

8. Aylward EH, Roberts-Twillie JV, Barta PE, Kumar AJ, Harris GJ, Geer $M$, et al. Basal ganglia volumes and white matter hyperintensities in patients with bipolar disorder. Am J Psychiatry 1994;151:687-93.

9. Dupont RM, Jernigan TL, Heindel W, Butters N, Shafer K, Wilson $\mathrm{T}$, et al. Magnetic resonance imaging and mood disorders. Localization of white matter and other subcortical abnormalities. Arch Gen Psychiatry 1995;52:747-55.

10. Elkis H, Friedman L, Wise A, Meltzer HY. Meta-analyses of studies of ventricular enlargement and cortical sulcal prominence in mood disorders. Comparisons with controls or patients with schizophrenia. Arch Gen Psychiatry 1995;52:735-46.

11. Swayze VWD, Andreasen NC, Alliger RJ, Ehrhardt JC, Yuh WT. Structural brain abnormalities in bipolar affective disorder. Ventricular enlargement and focal signal hyperintensities. Arch Gen Psychiatry 1990;47:1054-9.

12. Ahearn EP, Steffens DC, Cassidy F, Van Meter SA, Provenzale JM, Seldin MF, et al. Familial leukoencephalopathy in bipolar disorder. Am J Psychiatry 1998;155:1605-7.

13. Bilder RM, Wu H, Bogerts B, Ashtari M, Robinson D, Woerner $\mathrm{M}$, et al. Cerebral volume asymmetries in schizophrenia and mood disorders: a quantitative magnetic resonance imaging study. Int $\mathbf{J}$ Psychophysiol 1999;34:197-205.

14. Hirayasu Y, McCarley RW, Salisbury DF, Tanaka S, Kwon JS, Frumin M, et al. Planum temporale and Heschl gyrus volume reduction in schizophrenia: a magnetic resonance imaging study of first-episode patients. Arch Gen Psychiatry 2000;57:692-9.

15. Sax KW, Strakowski SM, Zimmerman ME, DelBello MP, Keck-Jr PE,
Hawkins JM. Frontosubcortical neuroanatomy and the continuous performance test in mania. Am J Psychiatry 1999;156:139-41.

16. Soares JC, Mann JJ. The functional neuroanatomy of mood disorders. J Psychiatr Res 1997;31:393-432.

17. Drevets WC, Price JL, Simpson Jr JR, Todd RD, Reich T, Vannie $\mathrm{M}$, et al. Subgenual prefrontal cortex abnormalities in mood disorders. Nature 1997;386:824-7.

18. Hirayasu Y, Shenton ME, Salisbury DF, Kwon JS, Wible CG, Fischer IA, et al. Subgenual cingulate cortex volume in firstepisode psychosis. Am J Psychiatry 1999;156:1091-3.

19. Altshuler LL, Conrad A, Hauser P, Li XM, Guze BH, Denikoff K, et al. Reduction of temporal lobe volume in bipolar disorder: a preliminary report of magnetic resonance imaging. Arch Gen Psychiatry 1991;48:482-3.

20. Bremner JD, Narayan M, Anderson ER, Staib LH, Miller HL, Charney DS. Hippocampal volume reduction in major depression. Am J Psychiatry 2000;157:115-8.

21. Sheline YI, Sanghavi M, Mintun MA, Gado MH. Depression duration but not age predicts hippocampal volume loss in medically healthy women with recurrent major depression. J Neurosci 1999;19:5034-43.

22. Altshuler LL, Bartzokis G, Grieder T, Curran J, Jimenez T, Leight K, et al. An MRI study of temporal lobe structures in men with bipolar disorder or schizophrenia. Biol Psychiatry 2000;48:147-62.

23. Strakowski SM, DelBello MP, Sax KW, Zimmerman ME, Shear PK, Hawkins JM, et al. Brain magnetic resonance imaging of structural abnormalities in bipolar disorder. Arch Gen Psychiatry 1999;56:254-60.

24. Krishnan KR, McDonald WM, Escalona PR, Doraiswamy PM, Na C, Husain MM, et al. Magnetic resonance imaging of the caudate nuclei in depression. Preliminary observations. Arch Gen Psychiatry 1992;49:553-7.

25. Husain MM, McDonald WM, Doraiswamy PM, Figiel GS, Na C, Escalona PR, et al. A magnetic resonance imaging study of putamen nuclei in major depression. Psychiatry Res 1991;40:95-9.

26. Pillay SS, Yurgelun-Todd DA, Bonello CM, Lafer B, Fava M, Renshaw PF. A quantitative magnetic resonance imaging study of cerebral and cerebellar gray matter volume in primary unipolar major depression: relationship to treatment response and clinical severity. Biol Psychiatry 1997;42:79-84.

27. DelBello MP, Strakowski SM, Zimmerman ME, Hawkins JM, Sax KW. MRI analysis of the cerebellum in bipolar disorder: a pilot study. Neuropsychopharmacol 1999;21:63-8.

28. Wu JC, Buchsbaum MS, Johnson JC, Hershey TG, Wagner EA, Teng $\mathrm{C}$, et al. Magnetic resonance and positron emission tomography imaging of the corpus callosum: size, shape and metabolic rate in unipolar depression. J Affect Disord 1993;28:15-25.

29. Dasari M, Friedman L, Jesberger J, Stuve TA, Findling RL, Swales TP, et al. A magnetic resonance imaging study of thalamic area in adolescent patients with either schizophrenia or bipolar disorder as compared to healthy controls. Psychiatry Res 1999;91:155-62.

\section{Correspondência: Jair C. Soares}

Neurochemical Brain Imaging Laboratory, Western Psychiatric Institute and Clinic, University of Pittsburgh School of Medicine 3811 O'Hara St., Pittsburgh, PA - 15213 USA

Fax: (00xx1) (412) 624-1496 - Email: soares+@ pitt.edu 